\title{
Postpartum atypical haemolytic uraemic syndrome in a pre-eclamptic patient
}

Efterpi Tingi $^{1} *$, Nada Sabir ${ }^{2}$, Lynda Verghese ${ }^{3}$

\author{
${ }^{1}$ Specialty trainee in Obstetrics and Gynaecology St Mary's Hospital, Oxford Road, Manchester, UK \\ ${ }^{2}$ Consultant Obstetrician and Gynaecologist, Bradford Teaching Hospitals, Bradford, UK \\ ${ }^{3}$ Consultant Obstetrician and Gynaecologist, Wrexham Maelor Hospital, Wales, UK
}

Received: 09 January 2016

Accepted: 06 February 2016

\author{
*Correspondence: \\ Dr. Efterpi Tingi \\ E-mail: efterpi.tingi@ doctors.org.uk
}

Copyright: (C) the author(s), publisher and licensee Medip Academy. This is an open-access article distributed under the terms of the Creative Commons Attribution Non-Commercial License, which permits unrestricted non-commercial use, distribution, and reproduction in any medium, provided the original work is properly cited.

\begin{abstract}
Postpartum atypical haemolytic uraemic syndrome (aHUS) is a rare manifestation, defined as non-immune haemolytic anaemia, thrombocytopenia, and renal thrombotic microangiopathy (TMA) typically following a normal vaginal delivery. Most cases of aHUS are caused by uncontrolled chronic activation of alternative complement pathway, resulting in microvascular thrombosis, organ ischaemia and damage. aHUS has a poor prognosis, hence up to 65 percent of patients require haemodialysis or have kidney damage or die. We report a case of postpartum atypical haemolytic uraemic syndrome in a 30 year old multiparous woman, who presented with orthopnoea, chest and abdominal pain, seven days following emergency caesarean section due to severe preeclampsia. Clinical examination and several investigations revealed anaemia, thrombocytopenia and renal failure, findings suggestive of thrombotic microangiopathy, therefore she was admitted in ITU where total plasma exchanged was initiated. The patient improved 10 days post ITU admission and discharged home. This case highlights that the TMAs may be indistinguishable clinically from each other, making a formal diagnosis difficult. Atypical HUS should be suspected during the postpartum period in any woman who presents with haeomolytic anaemia, thrombocytopenia and severe renal impairment. Aggressive treatment with plasma exchange may limit vascular injury and improve prognosis.
\end{abstract}

Keywords: Haemolytic uraemic syndrome, Pre-eclampsia, Pregnancy

\section{INTRODUCTION}

Postpartum atypical haemolytic uraemic syndrome (aHUS) is a rare manifestation with an incidence of 1 in 25000 pregnancies. $^{1}$ It is defined as non-immune haemolytic anaemia, thrombocytopenia, and renal thrombotic microangiopathy (TMA) typically following a normal vaginal delivery. ${ }^{2}$ Most cases of aHUS are caused by uncontrolled chronic activation of alternative complement pathway, resulting in microvascular thrombosis, organ ischaemia and damage. ${ }^{3}$ aHUS has a poor prognosis, hence up to 65 percent of patients require haemodialysis or have kidney damage of varying severity or die. Therefore a high index of suspicion and awareness of its clinical presentation is crucial to accurate diagnosis and management.

\section{CASE REPORT}

A 30 year old multiparous woman of Caucasian origin had been generally fit and well. She had a previous Caesarean Section for twins at 28 weeks gestation. She had regular antenatal care and developed preeclampsia at 33 weeks controlled with labetalol. She presented with persistent headaches, poorly controlled blood pressure despite treatment at $35+4$ week. Her blood pressure was stabilised and she received two doses of steroids to aid fetal lung maturity. Urine protein creatinine ratio was 193, creatinine (64), platelets 229x109/L, haemoglobin 
$12.4 \mathrm{~g} \%$. She underwent an uneventful lower segment caesarean section and she was discharged six days postsurgery on oral labetalol $200 \mathrm{mg}$ four times a day.

The following day which was the seventh postoperative day, she presented with severe chest pain, dyspnoea, orthopnoea, lethargy, and abdominal pain. The vital signs showed pulse of 49-70 bpm, respiratory rate of 24 breaths/ minute, blood pressure 165-180/88-100 mmHg. Clinical examination revealed pallor and generalized oedema. Chest and abdominal examination were unremarkable. Blood results on admission showed anaemia (Hb 7.3\%), thrombocytopenia (platelets $55 \times 109 / \mathrm{L}$ ) and raised creatinine (193 and peaked to 371 ), increased lactate dehydrogenase (LDH: $5000 \mathrm{UI} / \mathrm{ml})$, and normal liver function tests. Blood film was in keeping with schistocytosis and haemolysis. Intravenous labetalol and magnesium bolus and infusion were commenced. The clinical picture of microangiopathic haemolysis was evident but was it secondary to HELLP syndrome, aHUS or TTP. But since the clotting profile was normal and the renal impairment was predominantly significant, the provisional diagnosis was aHUS.

Following a sudden drop in GCS the patient was admitted in intensive care unit (ITU). Hypertension was managed with nifedipine, doxazocin and Atenolol. Plasma exchange was initiated. CT scan of the brain was normal. The patient improved 10 days post ITU admission and gradual improvement of LDH, platelets (289) and creatinine (207) were noted.

\section{DISCUSSION}

Haemolytic uraemic syndrome is a thrombotic microangiopathic disorder (TMA), defined as nonimmune haemolytic anaemia, thrombocytopenia and renal impairment, characterized by fragmented red blood cells (schistocytes) on peripheral blood smear, low haptoglobin levels, a decline in baseline hemoglobin, an elevated lactate dehydrogenase (LDH), and a negative direct Coombs assay. ${ }^{4}$ Most cases (more than 90\%) are associated with diarrhoea or shigan toxin producing bacteria or streptococci. Approximately, $10 \%$ of the cases are not associated with infections, hence they are characterised as atypical haemolytic uraemic syndrome. ${ }^{5}$ It is thought that aHUS is related to the genetic hyperactivation of the alternative complement cascade.

Pregnancy associated TMA is characterized by occurrence of fibrin and/or platelet aggregation in the microvasculature, resulting in mechanical haemolysis and thrombocytopenia during the ante partum or postpartum period. Its incidence is 1 in 25000 pregnancies. ${ }^{6}$ As many as $20 \%$ of women with aHUS experienced their first episode around the time of a pregnancy, with the majority of these $(80 \%)$ being in the postpartum period. ${ }^{7}$ TMA can also be associated with reduced activity of a disintegrin and metalloproteinase with thrombospondin type 1 motif, 13 (ADAMTS13). ADAMTS 13 is a cleavage molecule that acts upon ultra-large von Willebrand factors (vWF) and is related to the development of thrombotic thrombocytopenic purpura (TTP). ${ }^{8}$

There are two forms of aHUS, the familial form with less than $20 \%$ of cases and the sporadic form. The sporadic form develops in patients with different conditions which are associated with the development of TMA such as infections, HIV, malignancy, organ transplantation, pregnancy, catastrophic antiphospholipid syndrome (APLS), malignant hypertension, and the use of chemotherapeutic and antiplatelet agents (Table 1). ${ }^{9}$

Table 1. Direct causes and conditions associated with thrombotic microangiopathy.

\begin{tabular}{|c|c|}
\hline Direct causes of TMA & $\begin{array}{l}\text { Conditions associated } \\
\text { with TMA }\end{array}$ \\
\hline $\begin{array}{l}\text { Shiga-toxin E.coli (STEC- } \\
\text { HUS) }\end{array}$ & Malignancy \\
\hline $\begin{array}{l}\text { Abnormal cleavage of } \\
\text { vWF, ADAMTS } 13 \\
\text { deficiency (TTP) }\end{array}$ & Medications \\
\hline \multirow{5}{*}{$\begin{array}{l}\text { Uncontrolled complement } \\
\text { activation (aHUS) }\end{array}$} & Pregnancy \\
\hline & Surgery \\
\hline & Malignant hypertension \\
\hline & $\begin{array}{l}\text { Immune diseases (i.e. } \\
\text { antiphospholipid } \\
\text { syndrome) }\end{array}$ \\
\hline & Infections/HIV \\
\hline
\end{tabular}

Studies have shown that gene mutations encoding proteins which regulate the alternative pathway or antibodies that inhibit complement regulatory proteins are identified in $60-70 \%$ of patients with aHUS, ${ }^{10}$ whereas in the remaining patients there is not any identifiable genetic mutation. There are currently six important mutations responsible for dysregulated complement activation in aHUS patients, the complement factor $\mathrm{H}$ (CFH) mutations (23-27\%), membrane cofactor protein (MCP) (5\%), complement factor I (CFI) (4-8\%), and complement component C3 (C3) (2\%-8\%), respectively. Moreover, gene mutations in complement factor B (CFB; $1 \%-4 \%)$ and thrombomodulin (3\%-5\%) have also been noted. ${ }^{7}$ Mutations in complement genes predispose rather than causing aHUS. ${ }^{11}$

aHUS has a significant impact on renal function which may lead to the development of end stage renal disease (ESRD). Despite that, aHUS is a systemic disease with multi-organ and system involvement, including the cardiovascular system, ${ }^{11}$ gastrointestinal (diarrhoea \pm blood and/or, nausea/vomiting and/or abdominal pain and/or gastroenteritis), skin, and the central nervous system (confusion and/or seizures and/or other cerebral abnormalities). ${ }^{8}$

The diagnosis of aHUS should be based on clinical presentation and if there is not any identifiable cause of 
TMA, tests should be performed including full blood count, LDH, peripheral blood film, haptoglobin, renal and liver functions tests, as well as clotting times and fibrinogen concentrations. Moreover, some other tests should be considered when assessing patients with possible TMA include, ADAMTS 13, C3, C4, CFH and CFI serology, methylmalonic acid (urine and plasma), homocystine, HIV, ANA, lupus anticoagulant, antiphospholipid antibody, and direct antiglobulin. In addition, very young patients should be evaluated for streptococcal infection as a possible cause of their HUS. Genetic testing should be done early in suspected cases of aHUS and results should be waited before commencing treatment. $^{12}$

Table 2: Differential diagnosis of aHUS.
The differential diagnosis of aHUS during the postpartum period could be difficult as there are various TMA manifestations with same characteristics and overlapping symptoms. These include HUS, TTP, pre-eclampsia (PET) and HELLP syndrome. HELLP syndrome is a group of symptoms that occurs in pregnant women who have pre-eclampsia or eclampsia, who also develop signs of liver damage and abnormalities in blood clotting. It is characterised by haemolysis, elevated liver enzymes and low platelet count (Table 2).

\begin{tabular}{|c|c|c|c|c|c|c|c|}
\hline & MAHA & Thrombocytopenia & Coagulopathy & $\begin{array}{l}\text { Raised } \\
\text { BP }\end{array}$ & $\begin{array}{l}\text { Abdominal } \\
\text { symptoms }\end{array}$ & $\begin{array}{l}\text { Renal } \\
\text { impairment }\end{array}$ & $\begin{array}{l}\text { Neurological } \\
\text { symptoms }\end{array}$ \\
\hline PET & + & + & \pm & +++ & \pm & \pm & ++ \\
\hline HELLP & + & ++ & \pm & + & +++ & + & \pm \\
\hline TTP & ++ & +++ & & \pm & + & ++ & +++ \\
\hline HUS & + & ++ & \pm & ++ & + & +++ & \pm \\
\hline AFLP & \pm & + & ++++ & + & +++ & ++ & + \\
\hline SLE & + & + & \pm & + & \pm & ++ & + \\
\hline APLS & + & ++ & \pm & \pm & \pm & \pm & \pm \\
\hline
\end{tabular}

*MAHA: microangiopathic haemolytic anaemia, PET: pre-eclampsia HELLP (Haemolysis. EL (elevated liver) enzymes. LP (low platelet) count; TTP: thrombotic thrombocytopenic purpura; AFLP (Acute fatty liver of pregnancy); SLE: Systemic lupus erythematosus; APLS: Antiphospholipid syndrome.

Thrombotic thrombocytopenic purpura (TTP) is characterized by a pentad of thrombocytopenia, microangiopathic haemolytic anaemia (MAHA), fluctuating neurological signs, renal impairment and fever, often with insidious onset. However, TTP can present without the full pentad; up to $35 \%$ of patients do not have neurological signs at presentation and renal abnormalities and fever are not prominent features. The revised diagnostic criteria state that TTP must be considered in the presence of thrombocytopenia and MAHA. When there is diffuse vascular endothelial insult, endothelial cells secrete unusually large forms of vWF. In familial TTP, there is a constitutional deficiency of vWFcleaving protease while in non-familial TTP there is an inhibitor of vWF-cleaving protease (ADAMST-13).In HUS there is no deficiency. ${ }^{13}$

In summary, the presence of thrombocytopenia and microangiopathic anaemia and evidence of multi- organ damage like renal impairment, neurological symptoms, and/or gastrointestinal symptoms, indicates the use of the Shiga toxin test to exclude the diagnosis of STEC-HUS. The ADAMTS13 test should be used for the differentiation between TTP ( $\leq 5 \%$ activity) and aHUS (>5\% activity). ${ }^{8}$ While waiting for ADAMTS13 results, severe thrombocytopenia (a platelet count $>30 \times 109 / \mathrm{L}$ ) along with moderate renal failure (serum creatinine $>150$ $200 \mu \mathrm{moI} / \mathrm{L}(>1.7-2.3 \mathrm{mg} / \mathrm{dL})$, could be indicative of severe ADAMTS13 deficiency (TTP), whilst severe renal failure with platelets $>30 \times 109 \backslash \mathrm{L}$ would indicate aHUS. There is no evidence that delivery affects the course of
HUS and it is probable that failure for spontaneous recovery to occur after delivery of a pregnant patient with features of preeclampsia/HELLP syndrome suggests the ongoing microangiopathy is secondary to TTP/HUS. ${ }^{14}$

In adults, distinction between the two conditions aHUS or TTP is academic, as some women will develop neither neurologic symptoms nor renal disease but some develop both. Both conditions are managed with plasmapheresis and it has become common to use the term TTP/HUS complex to describe this condition. Ten to $25 \%$ of all TTP/HUS cases occur in pregnancy and it is likely that TTP/HUS are both on a continuum with preeclampsia and HELLP syndrome, as all conditions are characterised by widespread endothelial cell injury. ${ }^{14}$

Based on previous treatment guidelines for aHUS published in $2009,{ }^{15}$ the standard aHUS therapy is plasma exchange (PE) and plasma infusion (PI) at presentation during the first month of aHUS, in both adults and children. $^{15}$ After 5 days of daily PE, aHUS was considered resistant ${ }^{11}$ if the platelet counts were still below $150 \times 109$ or if serum creatinine had not decreased by $>25 \%$ or the haemolysis was persisting. Plasma exchange, ${ }^{16}$ is thought to be related to its ability to deliver normal levels of $\mathrm{CFH}, \mathrm{CFI}, \mathrm{CFB}$ and $\mathrm{C} 3$ and to remove mutant $\mathrm{CFH}, \mathrm{CFI}, \mathrm{CFB}, \mathrm{C} 3$ and anti $\mathrm{CFH}$ antibodies when plasma is exchanged by apheresis. PE decreases mortality by $50 \%$. Platelet transfusions are contraindicated. 
Furthermore, in 2011, U.S Food and Drug Administration (FDA), approved eculizumab to be introduced in aHUS treatment. Ecuzilumab, is a recombinant humanized monoclonal IgG2/4 antibody that selectively targets component $\mathrm{C} 5$ and inhibits the terminal portion of the complement cascade, blocking the cleavage to C5a and $\mathrm{C} 5 \mathrm{~b}$ but leaving the proximal functions of the complement active. ${ }^{17}$ Since its introduction in aHUS treatment, eculizumab has exhibited improved outcomes compared to plasma exchange and it could be introduced for non-response to plasma exchange or with transition the outpatient setting9. Recent studies have proven the efficacy of eculizumab for the restore renal function and prevention of further TMAs ${ }^{18,19}$ and several case reports have shown its significant role in the full recovery of neurological manifestations. ${ }^{17}$ Several patients with aHUS with either inadequate response to therapy or delayed diagnosis, will progress to end stage renal disease, therefore renal transplantation may be required.

Finally, the prognosis of aHUS is poor. Approximately up to $50 \%$ of aHUS patients' progress end-stage renal disease (ESRD) within a year despite plasma exchange or plasma infusion, and $25 \%$ die during the acute phase if it is not being managed appropriately. ${ }^{20}$ TMA may present again rapidly following renal transplant and is associated with a mortality rate of $7 \%$, and $50 \%$ graft failure within 5 years post-transplant.

\section{CONCLUSIONS}

The diagnosis of aHUS may be difficult since its presenting symptoms are common with other conditions like thrombotic thrombocytopenic purpura and preeclampsia with HELLP. Atypical HUS should be suspected during the postpartum period in any woman who presents with severe renal impairment, haemolytic anaemia, and thrombocytopenia. Disseminated intravascular coagulation (DIC) is also rare and prothrombin time is usually normal. Women with preeclampsia are more likely to have elevated hepatic transaminases, proteinuria and abnormal clotting. A high index of suspicion will help diagnose this condition earlier as it is really important to commence immediate treatment with plasma exchange, to avoid adverse outcomes.

\section{Funding: No funding sources Conflict of interest: None declared \\ Ethical approval: Not required}

\section{REFERENCES}

1. Fakhouri F, Roumenina L, Provot F, Sallée M, Caillard S, Couzi L et al. Pregnancy-associated hemolytic uremic syndrome revisited in the era of complement gene mutations. J Am Soc Nephrol. 2010;21(5):859-67.

2. Noris M, Remuzzi G. Hemolytic uremic syndrome. J Am Soc Nephrol. 2005;16:1035-50.
3. Kourouklaris A, Ioannou K, Athanasiou I, Panagidou A, Demetriou K, Zavros M. Postpartum thrombotic microangiopathy revealed as atypical hemolytic uremic syndrome successfully treated with eculizumab: a case report. J Med Case Reports. 2014;8:307.

4. Roumenina LT, Loirat C, Dragon-Durey MA, Halbwachs-Mecarelli L, Sautes-Fridman C, Fremeaux-Bacchi V. Alternative complement pathway assessment in patients with atypical HUS. J Immunoll Methods. 2011;365(1):8-26.

5. Noris M, Remuzzi P. Atypical haemolytic uraemic syndrome. N engl j med. 2009;361:17.

6. Dashe JS, Ramin SM, Cunningham FG. The longterm consequences of thrombotic microangiopathy (thrombotic thrombocytopenic purpura and hemolytic uremic syndrome) in pregnancy. Obstet Gynecol. 1998;91:662-8.

7. Nester CM, Thomas CP. Atypical hemolytic uremic syndrome: what is it, how is it diagnosed, and how is it treated? Hematology Am Soc Hematol Educ Program. 2012;617-25.

8. Kuypers D, Campistol J, Legendre C. Diagnostic challenges in thrombotic microangiopathies. Eur Med J. 2014;28-36.

9. Loirat C, Saland J, Bitzan M. Management of hemolytic uremic syndrome. Presse Med. 2012;41:115-35.

10. Noris M, Caprioli J, Bresin E, Mossali C, Pianetti G, Gamba $\mathrm{S}$ et al. Relative role of genetic complement abnormalities in sporadic and familial aHUS and their impact on clinical phenotype. Clin J Am Soc Nephrol. 2010;5(10):1844-59.

11. Noris M, Mescia F, Remuzzi G. STEC-HUS, atypical HUS and TTP are all diseases of complement activation. Nat Rev Nephrol. 2012;8:622-33.

12. Caprioli J, Noris M, Brioschi S, Pianetti G, Castelletti F, Bettinaglio $\mathrm{P}$ et al, Genetics of HUS: the impact of $\mathrm{MCP}, \mathrm{CFH}$, and IF mutations on clinical presentation, response to treatment, and outcome. Blood. 2006;108:1267-79.

13. Furlan M, Robles R, Galbusera M, Remuzzi G, Kyrle PA, Brenner B et al. Von Willebrand factorcleaving protease in thrombotic thrombocytopenic purpura and the haemolytic uraemic syndrome. N Eng J Med. 1998;339:1578-84.

14. Powrie RO, Greene MF, Camann W. deSwiet's medical disorders in obstetric practice. $5^{\text {th }}$ edition. Wiley-Blackwell. 2010.

15. Taylor CM, Machin S, Wigmore SJ, Tim HJ. Clinical practice guidelines for the management of atypical haemolytic uraemic syndrome in the United Kingdom. Br J Haematol. 2010;148:37-47.

16. Bell WR, Braine HG, Ness PM, Kickler TS. Improved survival in thrombotic thrombocytopenic purpura-haemolytic uremic syndrome: clinical experience in 108 patients. $\mathrm{N}$ Engl $\mathrm{J}$ Med. 1991;325:398-403. 
17. Ohanian M, Cable C, Halka K. Eculizumab safely reverses neurologic impairment and eliminates need for dialysis in severe atypical hemolytic uremic syndrome. Clin Pharmacol. 2011;3:5-12.

18. Legendre CM, Licht C, Muus P, Greenbaum LA, Babu S, Bedrosian $\mathrm{C}$ et al. Terminal complement inhibitor eculizumab in atypical hemolyticuremic syndrome. N Engl J Med. 2013;368:2169-81.

19. Fakhouri F et al. 2013; Abstract 5593 Presented at: ASN, 5-10 November 2013, Atlanta, USA

20. Zuber J, Fakhouri F, Roumenina LT, Loirat C, Frémeaux-Bacchi V, French study group for aHUS/C3G. Use of eculizumab for atypical haemolytic uraemic syndrome and C3 glomerulopathies. Nat Rev Nephrol. 2012;8:11:64357.

Cite this article as: Tingi E, Sabir N, Verghese L. Postpartum atypical haemolytic uraemic syndrome in a pre-eclamptic patient. Int J Reprod Contracept Obstet Gynecol 2016;5:894-8. 